\title{
Association of Self-Reported Weight Change and Quality of Life, and Exercise and Weight Management Behaviors Among Adults with Type 2 Diabetes Mellitus: The SHIELD Study
}

\author{
Susan Grandy, ${ }^{1}$ Kathleen M. Fox, ${ }^{2}$ and Debbra D. Bazata ${ }^{3}$ \\ ${ }^{1}$ AstraZeneca LP, Wilmington, DE 19850-5437, USA \\ ${ }^{2}$ Strategic Healthcare Solutions, LLC, Monkton, MD 21111-0543, USA \\ ${ }^{3}$ St. Luke's Primary Care South, Overland Park, KS 66213, USA \\ Correspondence should be addressed to Kathleen M. Fox, kathyfox@gforcecable.com
}

Received 2 December 2011; Accepted 17 February 2012

Academic Editor: Veselin Mitrovic

Copyright (c) 2012 Susan Grandy et al. This is an open access article distributed under the Creative Commons Attribution License, which permits unrestricted use, distribution, and reproduction in any medium, provided the original work is properly cited.

\begin{abstract}
Purpose. This study examined the association between self-reported weight change and quality of life, and exercise and weight management behaviors among individuals with type 2 diabetes mellitus (T2DM). Methods. In the US SHIELD study, respondents reported whether they had lost or gained weight compared with 1 year earlier and completed the SHIELD-WQ-9 quality of life questionnaire as well as provided information on their exercise and weight management behaviors in the past 12 months. Results. Sixteen percent of the respondents reported gaining weight $(n=460)$, and 30\% reported losing weight $(n=895)$. More respondents who reported losing weight exercised regularly, limited calorie and fat intake, and increased fiber, fruit, and vegetable intake compared with respondents who reported gaining weight $(P<0.01)$. For all nine aspects of daily life, a significantly greater proportion of respondents who reported losing weight reported improved well-being $(12 \%-44 \%)$ compared with respondents who reported gaining weight $(P<0.0001)$. Conclusions. Self-reported weight loss was associated with improved well-being, better exercise, and weight management behaviors among individuals with T2DM.
\end{abstract}

\section{Introduction}

During the past decade, there has been a dramatic increase in diabetes and obesity in the United States [1]. In the United States, there are 23.5 million adults aged 20 years or older with diabetes [2]. Worldwide, 366 million people have diabetes in 2011, with the number of people with type 2 diabetes mellitus (T2DM) increasing in every country [3]. The increasing prevalence of T2DM is directly related to an increasing rise in the prevalence of obesity and physical inactivity, with an estimated 97 million US adults being overweight or obese $[4,5]$. National surveys report that $66 \%$ of Americans are overweight (body mass index $\left.(B M I)=25.0-29.9 \mathrm{~kg} / \mathrm{m}^{2}\right)$, and $32 \%$ are classified as obese $\left(\mathrm{BMI} \geq 30 \mathrm{~kg} / \mathrm{m}^{2}\right) \quad[1,6,7]$. More than 1.1 billion adults worldwide are overweight, and 312 million are obese [8]. Approximately $40 \%-50 \%$ of patients with diabetes also are obese [9].
Weight loss has been associated with reduced insulin resistance and improvements in glycemia [9]. Weight management is a key self-management treatment for individuals with T2DM. The American Diabetes Association (ADA) Standards of Medical Care in Diabetes [10] and International Diabetes Federation [3] recommend weight loss for all overweight or obese individuals who have T2DM to assist in achieving and maintaining glycemic control. Despite the recommendations, information on health outcomes associated with weight change is often limited for individuals with T2DM. Cross-sectional studies among the general population have shown a clear inverse relationship with baseline weight or BMI and health-related quality of life (HRQOL) [11-13]. Studies examining the change in weight over time and HRQOL found that weight gain led to a reduction in HRQOL $[14,15]$, and in general, weight loss $(5 \%-10 \%)$ was associated with significant improvement in HRQOL, typically in physical functioning and bodily 
pain $[14,16-18]$. However, only one study examined this association among individuals with T2DM [19], where the results indicated that patients with diabetes who lost $>5 \%$ of their weight during the clinical trial had improved their HRQOL scores.

For observational studies, methodological difficulties in assessing the association between weight change and HRQOL include the selection of the HRQOL instrument (condition specific or generic) and the timing of the HRQOL assessment in relation to the weight change. If the HRQOL instrument does not refer to weight or weight change, respondents may answer based on other health conditions that affect their HRQOL, and the association with weight change may be confounded. If a generic health instrument is used and the weight change occurred in the past before the recall period of the instrument, respondents may not consider their weight or change in weight when answering the survey. The present study examined the association between self-reported weight change and well-being utilizing the SHIELD-WQ-9, a new questionnaire developed to link HRQOL assessment to change in weight.

\section{Methods}

The present investigation is a cross-sectional analysis of data from the Study to Help Improve Early evaluation and management of risk factors Leading to Diabetes. (SHIELD) assessing the association between weight change and HRQOL. SHIELD is a 5-year, survey-based study conducted to better understand patterns of health status, health behavior, and knowledge and attitudes of people living with diabetes and those with varying levels of cardiometabolic risk.

2.1. SHIELD Survey. SHIELD included an initial screening phase to identify cases of interest in the general population (e.g., diabetes mellitus), a baseline survey to follow up identified cases with a questionnaire about health status, health knowledge and attitudes, current behaviors and treatments, and annual follow-up surveys. A detailed description of the SHIELD methodology has been published previously $[20,21]$.

In brief, the screening survey was mailed in April 2004 to a stratified random sample of 200,000 US households, representative of the US population for geographic residence, household size and income, and age of head of household [22], identified by the Taylor Nelson Sofres National Family Opinion (TNS NFO) panel (Greenwich, CT). All TNS NFO surveys were voluntary, and no special incentives were provided. A response rate of $64 \%$ was obtained for the screening survey. The SHIELD study was approved by the Quorum Review Board.

A comprehensive baseline survey was mailed in September-October 2004 to a representative sample of individuals $(n=22,001)$ who were identified in the screening survey as having self-reported type 1 diabetes mellitus or type 2 diabetes mellitus, no diabetes, or being at risk for diabetes. Each respondent group was balanced to be representative of that segment of the population for age, gender, geographic region, household size, and income for the US population, and then a random sample from each group was selected and sent the baseline survey. A response rate of $72 \%$ was obtained for the baseline survey. The 2008 annual follow-up survey (response rate of 71\%) included the SHIELD-WQ-9 questionnaire on weight change and how the weight change affected nine aspects of daily life as well as the SF-12 quality-of-life questionnaire. Responses from the 2008 survey were analyzed and reported in this study.

2.2. Study Measures. Respondents were classified as having T2DM based upon their self-report of having been told by a doctor, nurse, or other healthcare professional that they had T2DM. Other cardiovascular comorbid conditions were identified in a similar manner, with respondents having been told by a healthcare professional that they had cholesterol problems, circulation problems of any kind, heart disease or heart attack, hypertension, or narrow or blocked arteries.

For weight change, respondents were asked to compare their current weight with their weight 1 year ago and to indicate if they had gained weight, lost weight, stayed the same, lost weight then gained it back, or gained weight but lost it again. Utilizing the SHIELD-WQ-9, respondents were then asked the following: "thinking about your weight change or lack of weight change over the past year, how did this change (or lack of change) affect you in the following areas? In the past year, my change or lack of change in weight had the following effects." Response categories were "worsen, improved, stayed the same, and not applicable." The 9 following areas were reported: (1) how I feel physically (physical health), (2) my interactions with family, (3) my work performance, (4) my interactions with coworkers and friends, (5) my social activities, (6) my daily activities, (7) my self-esteem, (8) how I feel emotionally (emotional health), and 9) my overall quality of life. The SHIELD-WQ-9 was developed to assess HRQOL specific to changes in weight using the same domains (e.g., physical health and mental health), similar to other HRQOL instruments like the SF-36 and SF- 12 .

Exercise behaviors were reported based upon responses to a general exercise question and the International Physical Activity Questionnaire (IPAQ). First, respondents were asked to describe their exercise routine as "currently exercise regularly" versus "currently exercise some" or "currently do not exercise". Additionally, the type and length of physical activity over the previous 7 days were assessed through the 7item IPAQ short form [23]. The IPAQ scores are categorized into 3 levels: (1) low or inactive, (2) moderate activity of at least 600 metabolic equivalent (MET) minutes/week (3 or more days of vigorous activity of at least 20 minutes per day or 5 or more days of moderate activity or walking of at least 30 minutes per day), and (3) high activity of a minimum of 1500 MET minutes/week (vigorous activity on at least 3 days or 7 days of any combination of walking and moderate or vigorous activities). Weight management behaviors included responses to "have you tried to lose weight during the past 12 months" (yes/no), "have you done anything to keep from gaining weight during the past 12 months" (yes/no), "how often do you weigh yourself" (times per month), "how many 
times a week do you usually eat at a fast food restaurant," "which of these are you currently trying to do" (trying most of the time or trying very hard/every day versus trying some/little of the time or do not try to do), limit calories, eat less fat, eat more fiber, limit amount of meat, eat more fruit, and eat more vegetables. Weight and height was self-reported at the time of the survey. Actual weight change was computed by subtracting weight as reported in the 2007 survey from weight reported in the 2008 survey.

2.3. Statistical Analysis. The proportion of respondents reporting weight loss or weight gain was computed for respondents with T2DM. Comparisons between T2DM respondents who reported losing weight and those who reported weight gain were made using chi-square tests. Statistical significance was set $a$ priori as $P<0.05$.

\section{Results}

A total of 3,000 respondents with T2DM responded to the 2008 survey, of which 2,969 respondents answered the self-reported weight change question. Of the 2,969 T2DM respondents, $15.5 \%$ reported gaining weight over the past year, $30.1 \%$ reported losing weight, $41.1 \%$ reported staying the same, and $13.2 \%$ reported fluctuating weight. The analysis included those respondents reporting weight gain $(n=460)$ or weight loss $(n=895)$ and excluded those not reporting weight change or consistent weight change.

Respondents who reported weight loss were similar to respondents who reported weight gain with regard to gender $(61 \%$ versus $64 \%$ women), race $(75 \%$ versus $71 \%$ white), household income, and education $(P>0.10)$ (Table 1). Additionally, cardiovascular comorbid conditions (e.g., cholesterol problems and heart disease) were similar between respondents who reported losing weight and those who reported gaining weight. However, those who reported weight loss were older (on average 3 years) and weighed less at the time of the survey than respondents who reported weight gain $(P<0.05)$. A greater proportion of respondents who reported weight gain were obese $\left(B M I \geq 30 \mathrm{~kg} / \mathrm{m}^{2}\right)$, compared with those who reported weight loss $(P<0.001)$. Using the reported weight in 2007 and 2008 to calculate weight change rather than self-reported weight change, $75 \%$ of respondents who reported losing weight actually did lose weight, an average of 9 pounds (Table 1). Similarly, 70\% of respondents who reported weight gain actually did gain weight, an average of 7 pounds. The correlation coefficient for the comparison of self-reported weight change with actual weight change was $0.56(P<0.001)$.

3.1. Exercise and Weight Management Behaviors. A greater proportion of respondents who reported losing weight $(30 \%)$ also reported exercising regularly compared with respondents who reported gaining weight $(18 \%, P<0.001)$ (Table 2). Likewise, more respondents who reported weight loss were highly active and fewer were inactive based on IPAQ scores, compared with respondents who reported weight gain $(P<0.0001)$. Positive weight management behaviors (tried to lose weight, keep from gaining weight) were more evident among respondents who reported weight loss compared with respondents who reported weight gain. A greater proportion of respondents reporting weight loss reported doing things to keep from gaining weight (78\%) compared with respondents who reported weight gain $(70 \%$, $P=0.001)$. Respondents who reported weight loss indicated they weighed themselves more frequently than respondents who reported weight gain; $35 \%$ of respondents with reported weight loss weigh themselves 6 or more times per month versus $24 \%$ of respondents with reported weight gain $(P=$ $0.001)$. Eating at fast food restaurants was more frequent among respondents who reported weight gain (20\% ate out 3 or more times per week) than respondents who reported weight loss $(11 \%, P=0.006)$. A greater proportion of respondents who reported weight loss indicated they had improved their diet by limiting calories, eating less fat, eating more fiber, limiting the amount of meat, and eating more fruits and vegetables, compared with respondents who reported weight gain $(P<0.01$ for each).

3.2. Well-Being with SHIELD-WQ-9. A significantly greater proportion of respondents who reported losing weight reported improved well-being for all 9 aspects of daily life compared with respondents who reported gaining weight (Table 3). Forty-four percent of respondents who reported losing weight reported improved physical health, compared with $3 \%$ of respondents who reported gaining weight $(P<$ $0.001)$. Similarly, $32 \%-39 \%$ of respondents who reported losing weight reported improved emotional health, selfesteem, and overall quality of life, compared with $5 \%$ of respondents who reported gaining weight $(P<0.001)$. Twenty-six percent of respondents who reported losing weight reported improvement in daily activities, compared with $4 \%$ of respondents who reported gaining weight $(P<$ 0.001 ). Likewise, $12 \%-18 \%$ of respondents who reported losing weight reported improvement in interactions with family, coworkers, and friends, work performance, and social activities, compared with $3 \%-5 \%$ of respondents who reported gaining weight $(P<0.001)$.

For respondents reporting weight loss, the greatest proportions indicating improvement were for physical health $(44 \%)$, self-esteem (39\%), and overall quality of life (34\%). Only $5 \%$ or less of the respondents reporting weight gain indicated improvement in any of the 9 aspects of daily life.

\section{Discussion}

Self-reported weight loss, compared with self-reported weight gain, was associated with improved well-being among individuals with T2DM. A significantly larger percentage of respondents reporting weight loss indicated improvement in all 9 aspects of daily life than respondents reporting weight gain. Additionally, respondents reporting weight loss had significantly better exercise and weight management behaviors. Respondents reporting weight loss exercised and were more physically active than respondents who reported weight gain. Respondents reporting weight loss indicated they managed their weight and diet better than respondents 
TABLE 1: Characteristics of SHIELD respondents with type 2 diabetes who reported gaining or losing weight in the past year.

\begin{tabular}{|c|c|c|}
\hline Characteristics & $\begin{array}{l}\text { Lost weight } \\
(n=895)\end{array}$ & $\begin{array}{l}\text { Gained weight } \\
\quad(n=460)\end{array}$ \\
\hline Age, years, mean (SD) & $63.0(11.8)^{*}$ & $60.0(10.6)$ \\
\hline Women, \% & 61.1 & 63.9 \\
\hline White, \% & 74.6 & 70.7 \\
\hline Income, $\%$ with $\leq \$ 35,000 /$ year & 34.9 & 36.1 \\
\hline $\begin{array}{l}\text { Education, \% with no more than a high school } \\
\text { degree }\end{array}$ & 31.7 & 36.4 \\
\hline Cholesterol problems, $\%$ & 73.5 & 76.7 \\
\hline Circulation problems of any kind, $\%$ & 19.9 & 23.0 \\
\hline Heart disease/heart attack, \% & 26.3 & 23.7 \\
\hline Hypertension, $\%$ & 73.0 & 76.7 \\
\hline Narrow or blocked arteries, $\%$ & 10.3 & 7.6 \\
\hline Weight, lbs, mean (SD) & $209.7(54.7)^{*}$ & $230.9(60.8)$ \\
\hline Body mass index (BMI) category, $\%$ & $*$ & \\
\hline Normal weight $\left(\mathrm{BMI}<25.0 \mathrm{~kg} / \mathrm{m}^{2}\right)$ & 11.8 & 3.8 \\
\hline Overweight $\left(\mathrm{BMI}=25.0-29.9 \mathrm{~kg} / \mathrm{m}^{2}\right)$ & 25.6 & 19.6 \\
\hline Obese $\left(\mathrm{BMI} \geq 30 \mathrm{~kg} / \mathrm{m}^{2}\right)$ & 62.6 & 76.7 \\
\hline $\begin{array}{l}\text { Calculated weight change from } 2007 \text { to } 2008 \text { in } \\
\text { pounds, mean (SD) }\end{array}$ & $-9.2(17.0)^{*}$ & $7.1(16.7)$ \\
\hline Lost weight from 2007 to $2008, \%$ & $75.4^{*}$ & 20.3 \\
\hline Gained weight from 2007 to $2008, \%$ & $15.8^{*}$ & 69.5 \\
\hline No change in weight from 2007 to $2008, \%$ & 8.8 & 10.2 \\
\hline
\end{tabular}

${ }^{*} P<0.05$ for comparison of lost weight versus gained weight; 1 pound $=0.4536 \mathrm{~kg}$.

reporting weight gain by monitoring their weight more frequently, trying to keep from gaining weight, eating less frequently at fast food restaurants, and eating healthier.

The evaluation of HRQOL in this study was performed using a newly developed, weight-related questionnaire (SHIELD-WQ-9). The SHIELD-WQ-9 was specifically developed to elicit HRQOL based on changes in weight, which may represent a truer impact of weight change on HRQOL than other general HRQOL instruments like the SF-36. Generic HRQOL instruments are not specifically targeted to weight change but overall quality of life, which most likely was influenced by more significant healthrelated issues than weight change, such as diabetes and/or cardiovascular disease, since $73 \%-77 \%$ of respondents had cholesterol problems and hypertension and 24\%-26\% had heart disease or heart attack. Further research is needed to validate the instrument in assessing weight-related HRQOL among individuals with diabetes as well as other health conditions.

This study adds to the evidence base for an association between weight loss and better HRQOL among individuals with T2DM. Very few studies have examined this relationship for adults with T2DM [19], and no study has been done on a large population-based sample of T2DM. The present study found a similar association of weight loss and improvement in HRQOL as other studies among the general population
$[14,16-18]$. With this evidence that weight loss is associated with improved HRQOL among individuals with T2DM, it is important to determine if the improved HRQOL along with the weight loss will assist T2DM patients to better manage their diabetes (possibly improve medication adherence and glucose monitoring) and control their glucose levels. Information about glycemic control (HbAlc levels) was not collected in the SHIELD survey; thus, this study was not able to investigate whether respondents who reported weight loss had better glycemic control. Future research is needed to further evaluate whether weight loss has a positive impact on diabetes self-management leading to better health outcomes.

Self-reported weight loss was also associated with better exercise and weight management behaviors. Respondents who reported weight loss also reported exercising more and eating better than respondents who reported weight gain. These lifestyle behaviors need to be encouraged and supported by family and the healthcare system so that adults with T2DM can successfully manage their weight and reduce the risk of obesity, which is at epidemic rates among individuals with T2DM. It is important to note that only $30 \%$ of respondents who reported weight loss indicated they exercised regularly and only $13 \%$ were highly active based on IPAQ scores. These figures indicate a large gap in lifestyle management even among those who 
TABLE 2: Exercise and weight management behaviors among respondents with type 2 diabetes who reported gaining or losing weight in the past year.

\begin{tabular}{|c|c|c|}
\hline & $\begin{array}{l}\text { Lost weight } \\
(n=895)\end{array}$ & $\begin{array}{l}\text { Gained weight } \\
\quad(n=460)\end{array}$ \\
\hline \multicolumn{3}{|l|}{ Exercise behavior } \\
\hline Currently exercise regularly, \% & $30.0^{*}$ & 17.7 \\
\hline International Physical Activity Questionnaire score & $*$ & \\
\hline Inactive/low activity & 62.7 & 73.8 \\
\hline Moderately active & 24.1 & 17.3 \\
\hline Highly active & 13.2 & 8.9 \\
\hline \multicolumn{3}{|l|}{ Weight management behavior } \\
\hline Tried to lose weight in past 12 months, $\%$ & 70.8 & 66.7 \\
\hline Done things to keep from gaining weight in past 12 months, \% & $78.2^{*}$ & 69.6 \\
\hline How often do you weigh yourself each month? & $*$ & \\
\hline 0 times per month & 15.1 & 25.4 \\
\hline 1 time & 18.5 & 21.7 \\
\hline $2-5$ times & 31.4 & 28.8 \\
\hline $6-15$ times & 12.8 & 10.7 \\
\hline 16 or more times & 22.3 & 13.4 \\
\hline Number of times per week that you eat at a fast food restaurant, $\%$ & * & \\
\hline 0 times per week & 42.4 & 33.7 \\
\hline 1 & 31.7 & 31.9 \\
\hline 2 & 14.8 & 14.1 \\
\hline $3-15$ & 11.1 & 20.3 \\
\hline \multicolumn{3}{|l|}{ Proportion who try to do the following most of the time or every day, $\%$} \\
\hline Limit calories & $50.7^{*}$ & 36.1 \\
\hline Eat less fat & $61.6^{*}$ & 50.7 \\
\hline Eat more fiber & $57.1^{*}$ & 47.6 \\
\hline Limit meat & $36.9^{*}$ & 30.3 \\
\hline Eat more fruit & $63.8^{*}$ & 51.4 \\
\hline Eat more vegetables & $68.2^{*}$ & 55.8 \\
\hline
\end{tabular}

${ }^{*} P<0.01$ for comparison of lost weight versus gained weight.

TABle 3: Proportion of respondents with type 2 diabetes who selfreported (SHIELD-WQ-9) improvement in quality of life over the past year related to their weight change.

\begin{tabular}{lcc}
\hline $\begin{array}{l}\text { Respondents indicating } \\
\text { improvement over the past year } \\
(\%)\end{array}$ & $\begin{array}{c}\text { Lost weight* } \\
(n=895)\end{array}$ & $\begin{array}{c}\text { Gained weight } \\
(n=460)\end{array}$ \\
\hline Physical health & 44.0 & 3.1 \\
Interactions with family & 17.6 & 5.3 \\
Work performance & 17.7 & 2.7 \\
Interactions with & 12.5 & 2.7 \\
coworkers/friends & 16.8 & 2.9 \\
Social activities & 25.5 & 3.6 \\
Daily activities & 39.4 & 4.9 \\
Self-esteem & 31.9 & 4.9 \\
Emotional health & 34.2 & 4.9 \\
Overall quality of life & &
\end{tabular}

reported weight loss. There is a large portion of respondents among those reporting weight loss who are not physically active or do not exercise regularly $(60 \%-70 \%)$. The gap in weight management is not as large, but only $51 \%-$ $62 \%$ of respondents reporting weight loss reported limiting calories, eating less fat, and eating more fiber, leaving 38\%$49 \%$ of respondents not adopting healthy lifestyle behaviors. These gaps in positive exercise and weight management behavior need to be narrowed so that greater improvements in HRQOL and other positive health outcomes can be experienced by more adults with T2DM.

There are limitations to the study that should be considered. The determination of T2DM and weight was made based upon self-report rather than clinical or laboratory measures. However, actual weight and weight change were captured, and the majority of respondents (75\%) did report their weight change accurately. The SHIELD-WQ-9 continues to be administered in different T2DM treatment settings to test its validity and reliability. Household panels, like the SHIELD study, tend to underrepresent the very wealthy 
and very poor segments of the population and do not include military or institutionalized individuals. However, these limitations are true for most random sampling and clinically based methodologies. Self-selection bias may be present because respondents were those who could read and comprehend the survey.

\section{Conclusions}

Self-reported weight loss, compared with self-reported weight gain, was associated with improved well-being and positive exercise and weight management behaviors among individuals with type 2 diabetes.

\section{Acknowledgments}

Members of the SHIELD Study Group are Harold Bays, MD, Louisville Metabolic and Atherosclerosis Research Center, Louisville, KY; Debbra D. Bazata, RD, CDE, St. Luke's Primary Care South, Overland Park, KS; James R. Gavin III, $\mathrm{MD}, \mathrm{PhD}$, Emory University School of Medicine, Atlanta, GA; Andrew J. Green, MD, Midwestern Endocrinology, Overland Park, KS; Sandra J. Lewis, MD, Northwest Cardiovascular Institute, Portland, OR; Michael L. Reed, PhD, Vedanta Research, Chapel Hill, NC; Helena W. Rodbard, MD Endocrine and Metabolic Consultants, Rockville, MD. Tina Fanning of Vedanta Research, Chapel Hill, NC, also contributed to this paper, performing data collection and analysis. Dr. S. Grandy is an employee and stockholder of AstraZeneca LP. Dr. K. M. Foxreceived research funds from AstraZeneca LP to conduct this study. This research was supported by AstraZeneca LP.

\section{References}

[1] A. H. Mokdad, B. A. Bowman, E. S. Ford, F. Vinicor, J. S. Marks, and J. P. Koplan, "The continuing epidemics of obesity and diabetes in the United States," Journal of the American Medical Association, vol. 286, no. 10, pp. 1195-1200, 2001.

[2] American Diabetes Association, "Diabetes statistics," http:// www.diabetes.org/diabetes-basics/diabetes-statistics.

[3] International Diabetes Federation., Diabetes Atlas, www. .diabetesatlas.org/.

[4] National Heart, Lung, and Blood Institute, "Clinical guidelines on the identification, evaluation, and treatment of overweight and obesity in adults: the evidence report," type 98-4083, NIH Publication, Bethesda, Md, USA, 1998.

[5] World Health Organization, "Global strategy on diet, physical activity, and health," http://www.who.intdietphysicalactivity/publications/facts.

[6] National Task Force on the Prevention and Treatment of Obesity, "Overweight, obesity, and health risk," Archives of Internal Medicine, vol. 160, no. 7, pp. 898-904, 2000.

[7] Centers for Disease Control, Prevalence of Overweight and Obesity Among Adults: United States. 2003-2004 http:// www.cdc.gov/nchs/products/pubs/pubd/hestats/overweight/ overwght_adult_03.htm.

[8] P. Hossain, B. Kawar, and M. El Nahas, "Obesity and diabetes in the developing world-a growing challenge," New England Journal of Medicine, vol. 356, no. 3, pp. 213-215, 2007.
[9] Centers for Disease Control, "Prevalence of overweight and obesity among adults with diagnosed diabetes, ," http://www .cdc.gov/mmwr/preview/mmwrhtml/mm5345a2.htm.

[10] American Diabetes Association, "Standards of Medical Care in Diabetes-2010," Diabetes Care, vol. 33, supplement 1, pp. S11S61, 2010.

[11] A. J. Lee, C. L. Morgan, M. Morrissey, K. U. Wittrup-Jensen, T. Kennedy-Martin, and C. J. Currie, "Evaluation of the association between the EQ-5Dindex (health-related utility) and body mass index (obesity) in hospital-treated people with Type 1 diabetes, Type 2 diabetes and with no diagnosed diabetes," Diabetic Medicine, vol. 22, no. 11, pp. 1482-1486, 2005.

[12] W. K. Redekop, M. A. Koopmanschap, R. P. Stolk, G. E. H. M. Rutten, B. H. R. Wolffenbuttel, and L. W. Niessen, "Healthrelated quality of life and treatment satisfaction in Dutch patients with type 2 diabetes," Diabetes Care, vol. 25, no. 3, pp. 458-463, 2002.

[13] L. S. Matza, N. Yurgin, K. S. Boye, K. Malley, and J. M. Shorr, "Obese versus non-obese patients with type 2 diabetes: patient-reported outcomes and utility of weight change," Current Medical Research and Opinion, vol. 23, no. 9, pp. 20512062, 2007.

[14] J. T. Fine, G. A. Colditz, E. H. Coakley et al., "A prospective study of weight change and health-related quality of life in women," Journal of the American Medical Association, vol. 282, no. 22, pp. 2136-2142, 1999.

[15] L. M. Leon-Munoz, P. Guallar-Castillon, J. R. Banegas et al., "Changes in body weight and health-related quality-of-life in the older adult population," International Journal of Obesity, vol. 29, no. 11, pp. 1385-1391, 2005.

[16] J. Kaukua, T. Pekkarinen, T. Sane, and P. Mustajoki, "Healthrelated quality of life in obese outpatients losing weight with very-low-energy diet and behaviour modification-A 2-y follow-up study," International Journal of Obesity, vol. 27, no. 10, pp. 1233-1241, 2003.

[17] B. Blissmer, D. Riebe, G. Dye, L. Ruggiero, G. Greene, and M. Caldwell, "Health-related quality of life following a clinical weight loss intervention among overweight and obese adults: intervention and 24 month follow-up effects," Health and Quality of Life Outcomes, vol. 4, p. 43, 2006.

[18] G. P. Samsa, R. L. Kolotkin, G. R. Williams, M. H. Nguyen, and C. M. Mendel, "Effect of moderate weight loss on healthrelated quality of life: an analysis of combined data from 4 randomized trials of sibutramine vs placebo," American Journal of Managed Care, vol. 7, no. 9, pp. 875-883, 2001.

[19] J. K. Kaukua, T. A. Pekkarinen, and A. M. Rissanen, "Healthrelated quality of life in a randomised placebo-controlled trial of sibutramine in obese patients with type II diabetes," International Journal of Obesity, vol. 28, no. 4, pp. 600-605, 2004.

[20] H. E. Bays, R. H. Chapman, and S. Grandy, "The relationship of body mass index to diabetes mellitus, hypertension and dyslipidaemia: comparison of data from two national surveys," International Journal of Clinical Practice, vol. 61, no. 5, pp. 737-747, 2007.

[21] H. E. Bays, D. D. Bazata, N. G. Clark et al., "Prevalence of self-reported diagnosis of diabetes mellitus and associated risk factors in a national survey in the US population: SHIELD (Study to Help Improve Early evaluation and management of risk factors Leading to Diabetes)," BMC Public Health, vol. 7, p. $277,2007$. 
[22] US Census Bureau, Annual Supplement to the Current Population Survey: Census Bureau Resident Population Estimates of the United States, US Census Bureau, Washington, DC, USA, 2003.

[23] C. L. Craig, A. L. Marshall, M. Sjöström et al., "International physical activity questionnaire: 12-Country reliability and validity," Medicine and Science in Sports and Exercise, vol. 35, no. 8, pp. 1381-1395, 2003. 


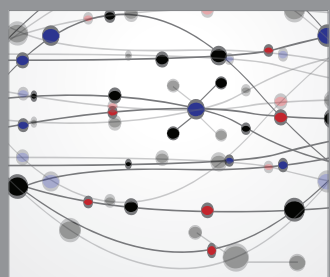

The Scientific World Journal
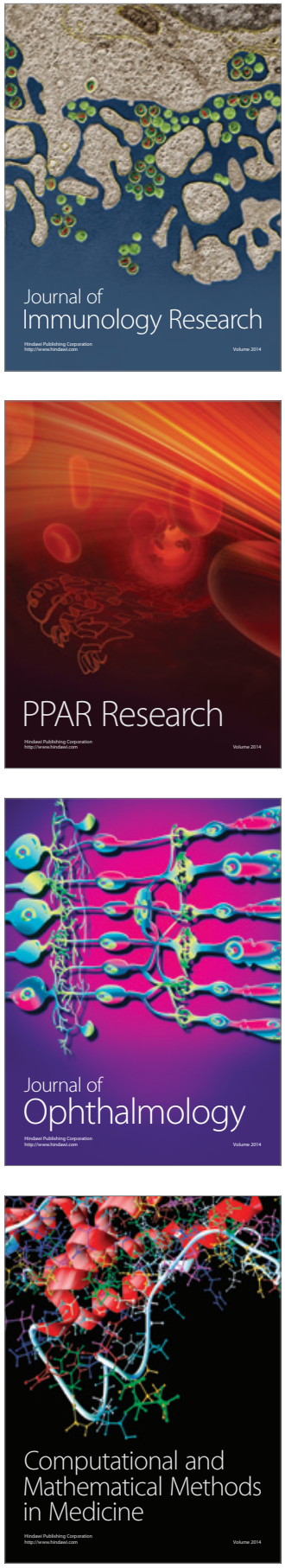

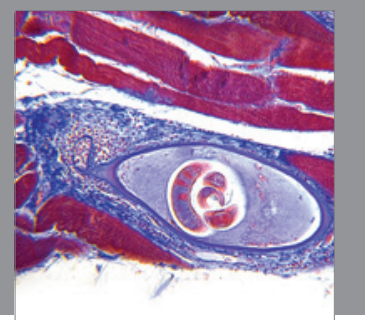

Gastroenterology

Research and Practice
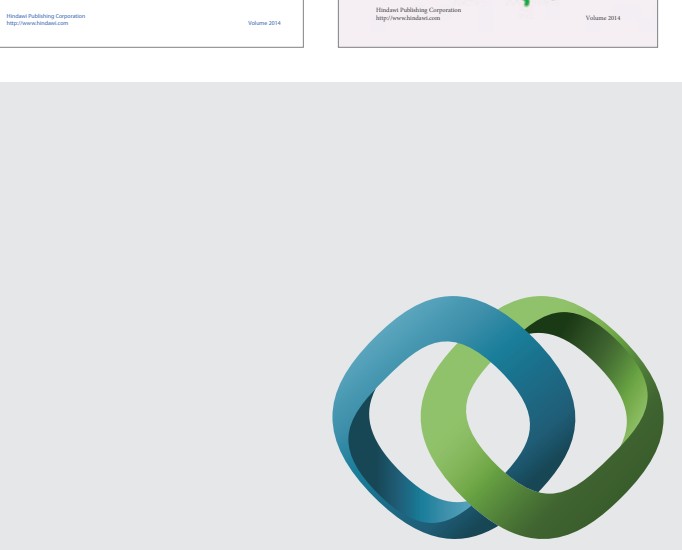

\section{Hindawi}

Submit your manuscripts at

http://www.hindawi.com
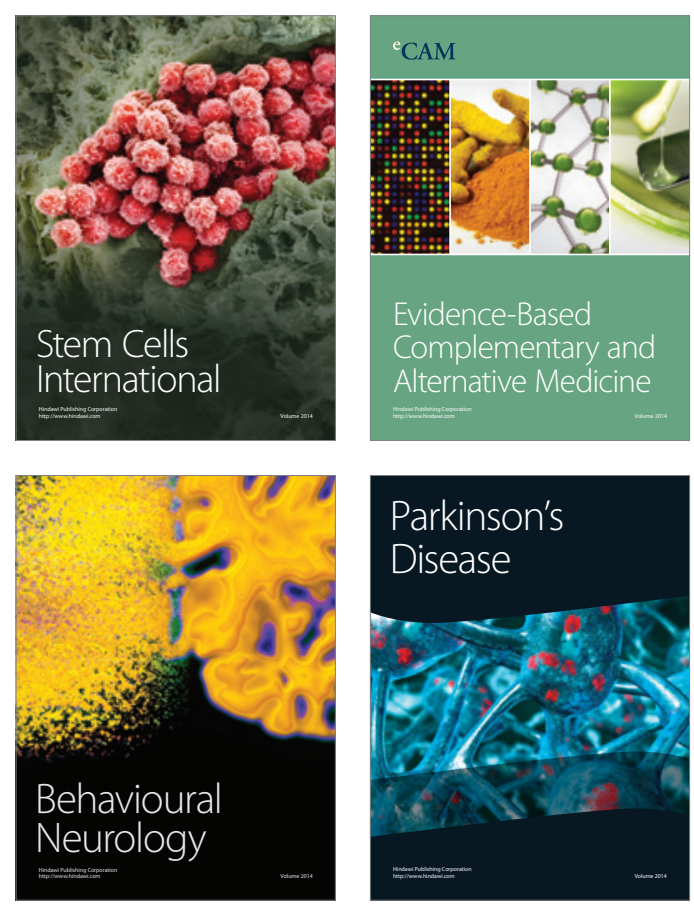

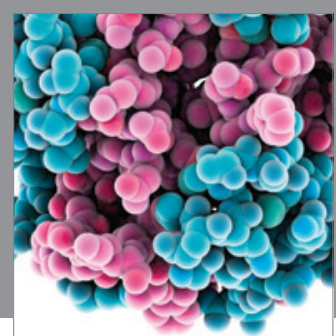

Journal of
Diabetes Research

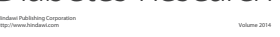

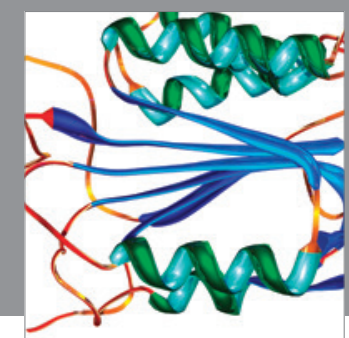

Disease Markers
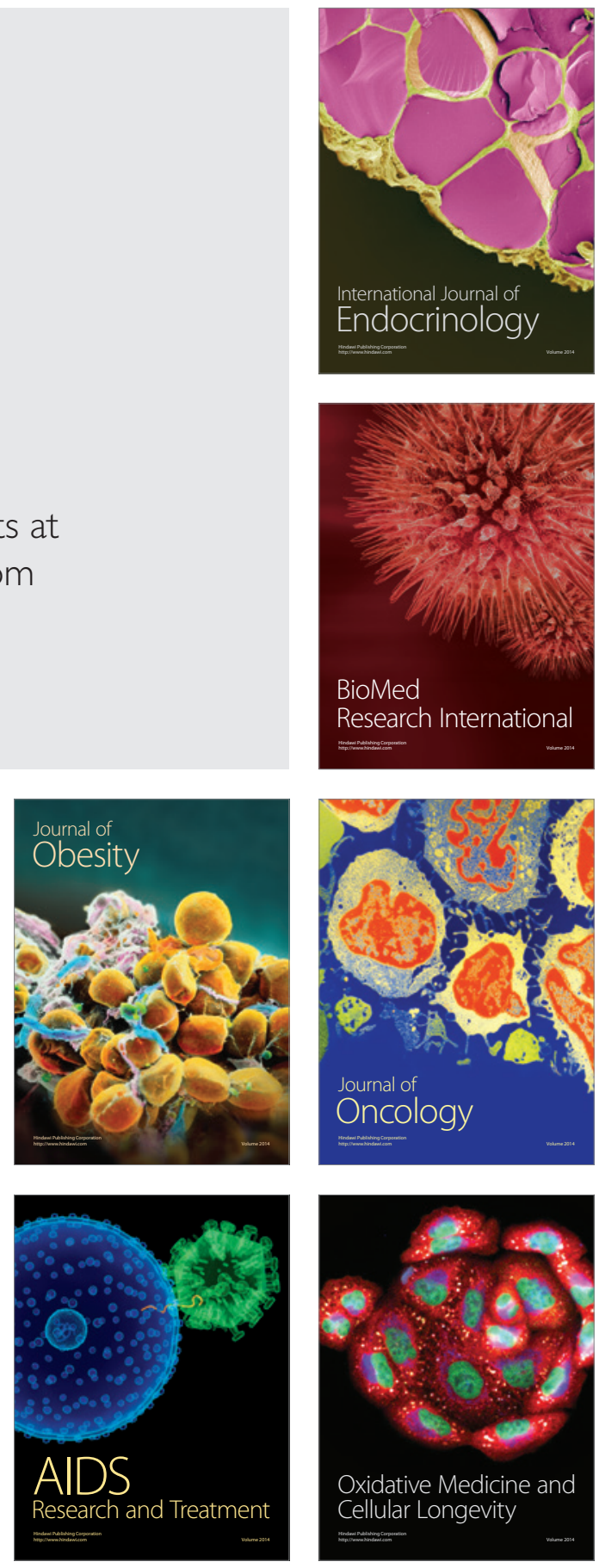